JOURNAL DE PHYSIQUE

Colzoque C6, supplément au n'12, Tome 42, décembre 1981

page $\quad 66-415$

\title{
ROLE OF POLAR PHONONS IN THE CHEMICAL BOUND AT STRUCTURAL PHASE TRANSITIONS CHARACTERIZED BY REPETITIVE FOURIER SPECTROSCOPY
}

\author{
F. Gervais and J.L. Servoin
}

Centre de Recherches sur la Physique des Hautes Températures, C.N.R.S., 45045 OrZéans, France

\begin{abstract}
The temperature dependence of dynamic effective charges carried by $T i$ and $\mathrm{Nb}$ ions which are the movable atoms involved in the ferroelectric phase transition of $\mathrm{BaTiO}_{3}$ and $\mathrm{KNbO}_{3}$, respectively, are reported, as deduced from TO-LO splittings of polar lattice modes, including soft modes. Results below the semiconducting-metal phase transition of $\mathrm{NbO}_{2}$ are also discussed.
\end{abstract}

There is an aspect of certain structural phase transitions with displacements of atoms, but which are not properly displacive, which has been little exploited up to now. When a structural transition occurs, certain cation-anion distances are significantly changed. Such changes of bond lengths affect not only the bond strength $\mathrm{k}$ and, therefore, the frequency of the oscillator $\omega_{0}=(k / \mu)^{1 / 2}$, but also the amount of the fractional charge which is supplied by the cation and localized on the anion. But things can be more complicated in compounds like oxides because oxygen becomes unstable, i.e. its polarisability increases rapidly with the increase of the radius of a spherical repartition of positive charges. ${ }^{1}$ The knowledge of instantaneous dipole momentscreated by the motion of positive against negative ions, by infrared reflectivity spectroscopy, gives information not only on polar optical modes and their splitting into TO and LO components, but also on the effective charge Ze. We will use

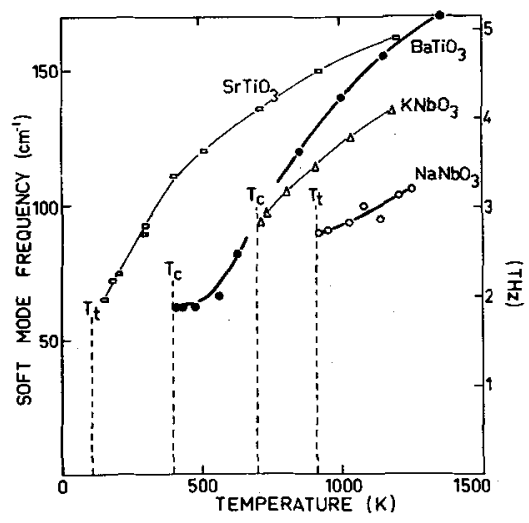

Fig. 1 : Temperature dependence of the soft mode frequency in the cubic $\mathrm{PE}$ phase of oxidic perovskites. the charge $z e$ introduced by scott, ${ }^{2}$ which is related to the szigeti effective charge $e_{s}$ via $z e=e_{s}\left(\varepsilon_{\infty}+2\right\} / 3 \varepsilon_{\infty}^{1 / 2}$, where $\varepsilon_{\infty}$ is the high frequency dielectric constant. Ze is the dynamic effective charge and incorporates the effects of polarisabilities and local fields. The dynamic effective charge has been measured in homopolar crystals such as graphite ${ }^{3}$ for example, and found to be finite wh reas the static charge is obviously zero. The TO and LO frequencies as derived by Cochran, 4 are thus rewritten

$$
\begin{aligned}
& \Omega_{\text {TO }}^{2}=k / \mu-(\mathrm{Ze})^{2} / 3 \mu \varepsilon_{\mathrm{v}} \mathrm{V} \\
& \Omega_{\mathrm{LO}}^{2}=\mathrm{k} / \mu+2(\mathrm{Ze})^{2} / 3 \mu \varepsilon_{\mathrm{v}} \mathrm{V}
\end{aligned}
$$



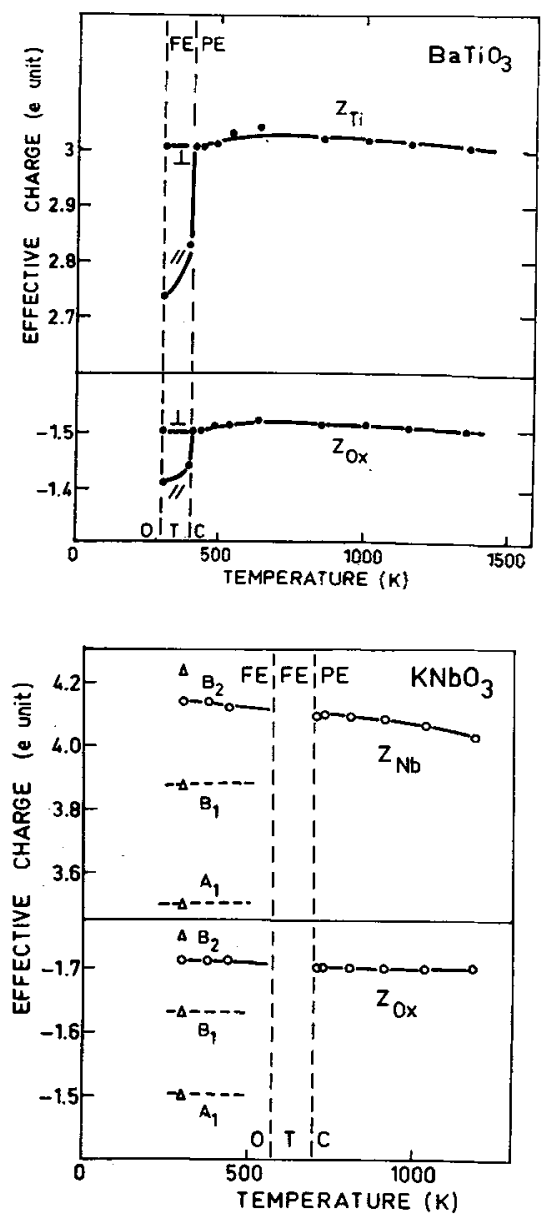

Fig. 2 : Temperature dependence of effective charges in $\mathrm{BaTiO}_{3}$ and $\mathrm{KNbO}_{3}$ with $\mathrm{z}_{\mathrm{Ba}}=1.5$ and $\mathrm{z}_{\mathrm{K}}=1$.
(2) minus (1) yielas

$$
\Omega_{L O}^{2}-\Omega_{\text {TO }}^{2}=(\mathrm{Ze})^{2} / \mu \varepsilon_{\mathrm{V}} \mathrm{V}
$$

a relation which has been generalized by scott ${ }^{2}$ in the form

$$
\sum_{j}\left(\Omega_{j L O}^{2}-\Omega_{j T O}^{2}\right)_{\alpha}=\left(\varepsilon_{v} v\right)^{-1} \sum_{k}(z e)_{k}^{2} / m_{k}
$$
where $\varepsilon_{v}$ is the dielectric constant of vacuum and the sum on the right side is over all $\mathrm{k}$ ions of mass $\mathrm{m}_{\mathrm{k}}$ in the elementary volume $v$. The temperature dependence of effective charges along a polarization $\alpha$ can thus be accurately deduced from that of TO and Lo frequencies. In strongly polar crystals such as oxidic perovskites, the contribution of the heavily damped soft To mode to Eq. (4) amounts to $\leqslant 18$ that of the weakly damped high-Exequency Lo mode in the cubic phase just above the temperature of transition to the tetragonal phase. It is, therefore, reasonable to renormalize the frequencies in Eq. (4), which has been derived in the harmonic approximation, to account for anharmonic corrections. Results for the temperature dependence of the soft mode in the cubic PE phase of $\mathrm{SrTiO}_{3}, \mathrm{BaTiO}_{3}, \mathrm{KNbO}_{3}$ and $\mathrm{NaNbO}_{3}$ as obtained by the authors and coworkers, ${ }^{5-8}$ are summarized in Fig. 1. Data have been deduced from i.r. reflection spectra recorded with a repetitive Fourier spectrometer and analyzed with the factorized form of the dielectric function. In such a system, the frequency accuracy is much better -

which is most important in Eq. (4) - than in conventional spectrometers since calibrated by comparison with that of a He-Ne laser and reaches $0.02 \mathrm{~cm}^{-1}$. It was found that a crossover from a regime which has the classical displacive soft mode behavior to a regime whose features are better described in the language traditionnally reserved for order-disorder systems, ${ }^{10}$ occurs above the temperature of the cubic - tetragonal transition so that this is finally not the soft mode which triggers the phase transition but rather hopping motions of ions in a triple-well potential. The displacement of $T i$ ion with respect to the oxygen octahedron, which occuxs at $T_{C}$ in $\mathrm{BaTiO}_{3}$ is however visualized in Fig. 2 on inspection of the marked and sudden decrease of $\mathrm{z}_{\mathrm{Ti}}$ and $\mathrm{z}_{\mathrm{Ox}}$ which is ascribed to the shortening of the $\mathrm{Ti}-\mathrm{O}$ length along the FE axis. Perpendicular to the direction of spontaneous polarization, no significant change of effective charge is observed over a wide temperature range, consis- 


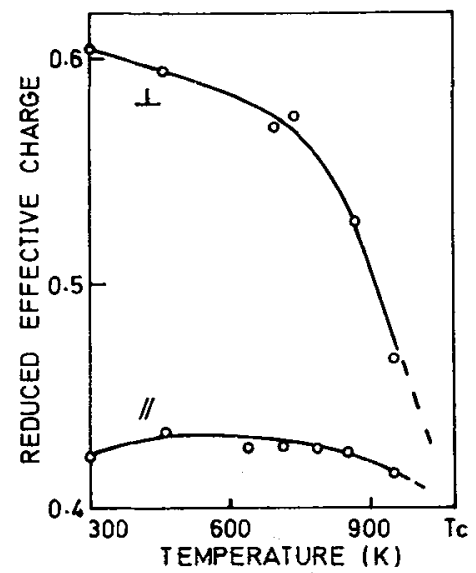

Fig. 3 : Effective charges in Nbo, measured parallel (//) and perpendicular $(\perp)$ to the airection of $\mathrm{Nb}-\mathrm{Nb}$ pairing. tent with the fact the $T i$ lons occupy the centrosymmetric site of the plane in both phases, at least with the largest probability. The same is found in $\mathrm{KNbO}_{3}$ perpendicular to the direction of spontaneous polarization ( $B_{2}$ modes) in the orthorhombic FE phase by comparison with the cubic PE phase as shown in Fig. 2, whereas significant decreases of charges have occured in other directions where certain $\mathrm{Nb}$ - O distances are thus confirmed to be shorter, in the FE phase. Let us briefly recall that this method, which, therefore, turns out to constitute a sensitive probe of (even small) ionic displacements, have also been successfully applied to the cases of ferroelectric $\mathrm{LiTaO}_{3}$ and $\mathrm{LiNbO}_{3},{ }^{12}$ and niobium dioxide. ${ }^{13}$ In the latter case, the low-temperature phase is essentially characterized by pairing of $\mathrm{Nb}$ atoms

two by two along the $c$ axis. Such a pairing reduces the effective charge $z / /$ in this direction with respect to $\mathrm{z}_{\perp}$ as shown in Fig. 3. $\mathrm{z} / /$ would increase and tend towards $z_{\perp}$ at the temperature of transition to the high-temperature rutile phase which occurs at $1080 \mathrm{~K}$ (in agreement with the observation of isotropic charges in other rutile oxide compounds) but this increase is masked by a decrease of $\sim 30 \%$ of both charges which is understood as a consequence of the progressive delocalization of electrons consistent with a change from semiconducting to metallic character which occurs at the $1080 \mathrm{~K}$ transition.

\section{References}

1. A. Bussmann, H. Bilz, R. Roenspiess and K. Schwarz, Ferroelectrics, $\underline{25}, 343$ (1980).

2. J.F. Scott, Rev. Mod. Phys., 46, 83 (1974).

3. R.J. Nemanich, G. Lucovsky and S.A. Solin, Solid St. Commun. 23, 117 (1977).

4. W. Cochran, Adv. Phys., 9, 387 (1960); 10, 401 (1961).

5. J.L. Servoin, Y. Luspin and F. Gervais, Phys. Rev. B22, 5501 (1980).

6. Y. Luspin, J.I.' Servoin and F. Gervais, J. Phys. C, $\overline{13}, 3761$ (1980).

7. A.M. Quittet, J.I. Servoin and F. Gervais, J. de Physique,42, 493 (1981). M.D. Fontana, G. Métrat, J.L. Servoin and F. Gervais, Ferroelectrics to be published).

8. F. Gervais, J.I. Servoin, J.F. Baumard and F. Denoyer, Solid St. Commun. (to be published).

9. K.A. Müller, Y. Luspin, J.L. Servoin and F. Gervais, (to be published).

10. A.D. Bruce, in "Solitons and Condensed Matter Physics", Ed. A.R. Bishop and T. Schneider, Springer Verlag (Berlin) p116 (1978).

11. Y. Luspin, J.L. Servoin, F. Gervais and A.M. Quittet, in "Symmetries and Broken Symmetries in Condensed Matter Physics", Ed. N. Boccara, Paris (1981), to be published.

12. F. Gervais, Phys. status Sol. (b), 100, 337 (1980).

13. F. Gervais, Phys. Rev.B23, 15 June issue (1981). 